\title{
Educação médica em tempos de crise: a experiência de uma liga acadêmica de dermatologia durante a pandemia da Covid-19
}

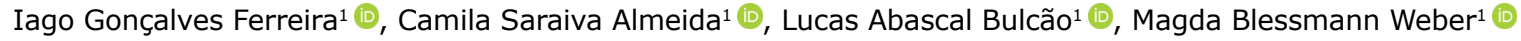

\section{RESUMO}

Introdução: A pandemia da Covid-19 impôs um grande desafio para as universidades ao redor do mundo, em meio a necessidade de distanciamento social causado pela disseminação do novo coronavírus (Sars-Cov-2). Frente a esta conjuntura, docentes e discentes foram instigados a buscar alterativas para continuidade das atividades universitárias, dentre as quais a adoção de Tecnologias de Informação e Comunicação, assim como, a ampliação do papel das atividades extracurriculares. Nessa perspectiva, este artigo teve como objetivo relatar a experiência da Liga Acadêmica de Dermatologia da Universidade Federal de Ciências da Saúde (Liaderm - UFCSPA) durante a pandemia da Covid-19, analisando a importância das atividades extracurriculares e da educação digital como ferramentas de Educação Médica. Relato de experiência: Durante o período de isolamento social e de suspensão de aulas, as atividades de ensino, pesquisa e extensão da Liaderm - UFCSPA precisaram ser readaptadas. Os encontros da liga, antes presenciais, passaram a ocorrer sob a forma de reuniões online em plataformas de videoconferência, como Zoom $®$ e Google Meet ${ }^{\circledR}$, nos quais eram organizadas aulas teóricas e discussão de casos clínicos. Aulas abertas à comunidade acadêmica também foram realizadas, como o webinar sobre Cirurgia Dermatológica, que teve participação de estudantes de medicina e outras áreas da saúde de todo o país. Além disso, em vista a manter o engajamento social dos membros, a Liaderm organizou um manual com orientações básicas acerca das lesões dermatológicas desencadeadas pelo uso de Equipamentos de Proteção Individual (EPIs), essenciais para a proteção dos profissionais de saúde envolvidos com o atendimento aos pacientes com Covid-19. Conclusões: Durante a pandemia, a maioria das atividades desenvolvidas pela Liaderm - UFCSPA pôde ser mantida mediante a adoção de Tecnologias de Informação e Comunicação, como as plataformas de videoconferência. Ademais, o alcance das atividades de ensino da liga foi expandido com a participação de acadêmicos de diferentes estados do país e do exterior. Nesse sentido, as adaptações provocadas pela pandemia podem repercutir a longo prazo, tanto nos modelos de ensino adotados pelas escolas médicas, quanto no reconhecimento das atividades extracurriculares como recursos educacionais estratégicos para a formação em Medicina.

Palavras-chave: Covid-19, Educação médica, Educação de graduação em medicina, Estudantes de medicina, Telemedicina.

1. Universidade Federal de Ciências da Saúde de Porto Alegre 


\section{INTRODUÇÃO}

O surgimento de uma nova cepa de coronavírus (Sars-CoV-2), em dezembro de 2019 em Wuhan na China, mobilizou diversos países e organismos internacionais. A doença causada por este agente foi denominada pela Organização Mundial de Saúde (OMS) Covid-19 e declarada Emergência de Saúde Pública de Importância Internacional em 30 de janeiro de 2020(1-3). Desde então, a Covid-19 disseminou-se por diversos países e continentes, adquirindo proporções e impactos inimagináveis.

Frente a esse cenário, as universidades foram fortemente impactadas necessitando interromper suas atividades de ensino tradicionais, como palestras e seminários, devido aos riscos aumentados de transmissão da doença, bem como a dificuldade em manter o distanciamento social adequado nas salas de aula(4-6). Dessa maneira, docentes e discentes foram expostos ao desafio de manter as atividades de estudo e pesquisa em meio a uma pandemia global.

Nessa perspectiva, as Tecnologias de Informação e Comunicação (TICs) reforçaram-se como uma valiosa ferramenta pedagógica, possibilitando a interação de docentes e estudantes através de tecnologias digitais como computadores, internet, aplicativos e smartphones, de maneira a estimular a participação ativa e colaborativa dos discentes(7). Todavia, o reconhecimento das possibilidades da educação à distância não é novidade, sendo difundido nas universidades desde o início do século XXI, impulsionado principalmente por suas vantagens como possibilidade de adaptação, disponibilidade de tempo, local e ritmo dos alunos, capacidade de alcance a regiões distantes dos grandes centros urbanos e pelos recursos de multimídia oferecidos(8).

Além das TICs, destacam-se também as atividades extracurriculares (AEs), iniciativas que propiciam uma formação profissional mais abrangente, a partir de experiências educacionais, além daquelas previstas pelo currículo universitário formal(9). Na graduação médica, as AEs podem ser desenvolvidas sob diversas formas: estágios ambulatoriais e hospitalares, iniciação científica, projetos de extensão, dentre outras $(10,11)$.

As ligas acadêmicas - entidades estudantis dedicadas ao estudo de temáticas específicas destacam-se entre as diversas modalidades de AEs devido a sua rica diversidade de experiências ofertadas, como aulas teóricas, cursos, simpósios, além de projetos de pesquisa e de extensão(10-12). Dessa maneira, estas associações têm o potencial de promover a autonomia, a proatividade e a consciência social dos estudantes $(10,11)$, atributos fundamentais para o processo de ensino e aprendizagem, principalmente durante a pandemia da Covid-19.

Diante desta conjuntura, este artigo tem como objetivo relatar as experiências de uma liga acadêmica de dermatologia durante a pandemia da Covid-19, analisando a importância que as atividades extracurriculares e a educação digital podem exercer na formação médica, principalmente frente à complexa conjuntura atual.

\section{RELATO DE EXPERIÊNCIA}

\section{A Liga Acadêmica de Dermatologia}

A Liga Acadêmica de Dermatologia da Universidade Federal de Ciências da Saúde de Porto Alegre (Liaderm - UFCSPA) é um projeto de extensão universitária do curso de Medicina, criado em 2014, com o objetivo de contribuir com o ensino da Dermatologia durante a graduação. Por meio de atividades acadêmicas baseadas no pilar ensino, pesquisa e extensão, esta entidade busca aproximar discentes interessados em Dermatologia com os conteúdos e a prática da especialidade.

$O$ ingresso de estudantes na Liaderm ocorre por seleção anual, na qual os candidatos são avaliados acerca de seu envolvimento com atividades extracurriculares, de suas habilidades no uso de tecnologias, e de seu interesse em relação à especialidade. $O$ processo seletivo ocorre através de entrevistas com membros veteranos da liga, e possibilita o ingresso de discentes tanto do curso de Medicina, de qualquer instituição de ensino superior, quanto de outras áreas da saúde da UFCSPA.

No ano de 2020, a Liaderm - UFCSPA era formada por 17 membros discentes: 15 membros do curso de Medicina, pertencentes a quatro universidades distintas, e dois do curso de Enfermagem da UFCSPA. As atividades ocorriam sob a orientação de uma docente do Departamento de Dermatologia da UFCSPA e um residente de dermatologia da UFCSPA-Santa Casa. 


\section{Atividades da liga anteriores à pandemia}

A Liaderm- UFCSPA, habitualmente, realizava seus encontros científicos de forma presencial com a participação de membros da liga e da docente coordenadora da Liga. Os encontros apresentavam frequência quinzenal, às terças-feiras, com duração de uma hora e meia, em sala de aula da universidade.

As aulas e discussões ocorriam a partir da apresentação de casos clínicos de doenças dermatológicas - mais prevalentes na prática médica - por dois alunos, seguidos de revisão teórica sobre a dermatose apresentada. Ao longo da sessão, os participantes eram instigados a formular hipóteses diagnósticas ao caso e, posteriormente, discutir o tema junto à coordenadora da liga. Além disso, eram realizados também encontros presenciais complementares abertos à comunidade acadêmica, através de aulas ministradas por dermatologistas da UFCSPA ou dermatologistas convidados.

Em relação às atividades de pesquisa, estas ocorriam frequentemente em parceria com o Serviço de Dermatologia da Santa Casa de Misericórdia de Porto Alegre / UFCSPA, mediante orientação da docente coordenadora da Liaderm. Em dezembro de 2019, uma parte dos membros esteve envolvida com a aplicação de questionários do Escore de Qualidade de Vida em Dermatologia Infantil (CDLQI), que avalia a qualidade de vida de pacientes com doenças dermatológicas. Ademais, os integrantes da liga eram convidados a participar de estudos de relatos de casos clínicos, sob instrução dos dermatologistas ou residentes do serviço da Santa Casa de Misericórdia de Porto Alegre/UFCSPA, possibilitando a integração e participação destes em jornadas e congressos de Dermatologia.

As iniciativas de extensão comunitária da liga eram desenvolvidas sob a forma de eventos abertos à comunidade e projetos direcionados a populações específicas. O grupo de apoio aos pacientes com dermatite atópica ("DermAlegria") consistia em uma das modalidades de extensão, por meio da qual eram realizados encontros mensais com rodas de conversa entre pais e pacientes pediátricos com dermatite atópica abordando a experiência pessoal destes com a doença. Algumas iniciativas eram desenvolvidas anualmente como o evento "UFCSPA Acolhe", nos meses de maio, em que a universidade abria suas portas para apresentar seus cursos, estrutura e atividades de extensão à comunidade. Neste evento, a Liaderm organizava oficinas ou capacitações sobre situações relevantes para a prática dermatológica, tendo como exemplo, a capacitação sobre automaquiagem corretiva para pessoas com doenças dermatológicas estigmatizantes, como vitiligo, realizada em 2019.

Além disso, os membros da liga participam anualmente do "Dezembro Laranja", campanha nacional de prevenção ao câncer de pele capitaneada pela Sociedade Brasileira de Dermatologia.

\section{Atividades da liga durante a pandemia da Covid-19}

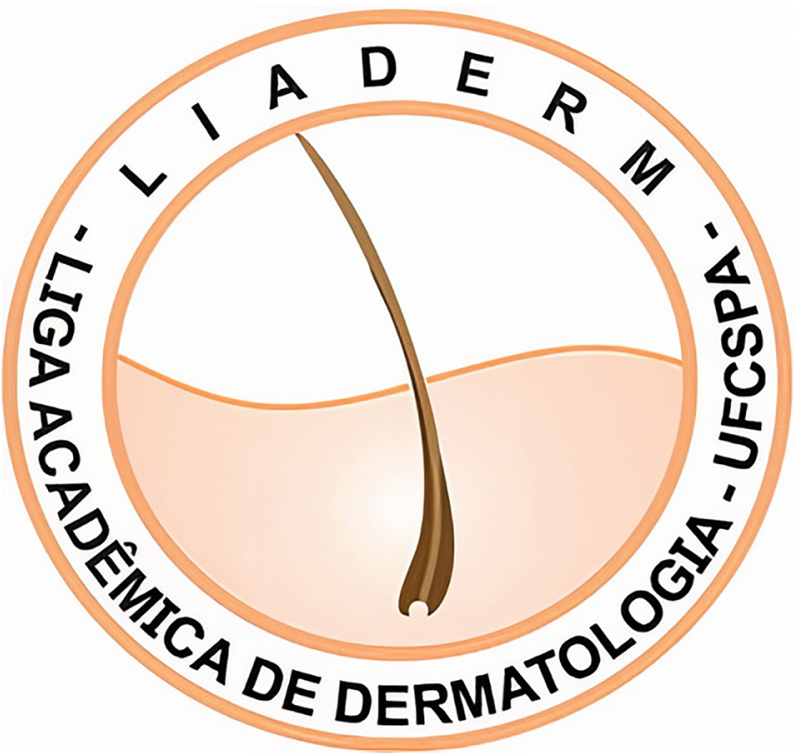

Figura 1 Logotipo da Liga Acadêmica de Dermatologia da UFCSPA.

Devido à necessidade de conter o avanço da pandemia e garantir a segurança dos alunos, a UFCSPA suspendeu o calendário acadêmico em 16 de março de 2020, cancelando também todas as atividades extracurriculares universitárias. Frente a essa conjuntura, a Liaderm optou por continuar com suas atividades mesmo à distância, de forma a atender à vontade de seus membros em manter os encontros e envolvimento com os conteúdos de Dermatologia.

\section{Aulas virtuais em plataformas de videoconferência}


A impossibilidade de realização de atividades presenciais, oriunda da necessidade de distanciamento social, tornou imperativa a busca por novas soluções para a manutenção das atividades da Liga. Assim, encontros virtuais em plataformas de videoconferência como Zoom $\AA$ e Google Meet $\AA$ foram a alternativa adotada.

Os encontros online apresentavam frequência semanal e duração de cerca de duas horas, ocorrendo sob a forma de casos clínicos dermatológicos e aulas teóricas ministradas por docentes dermatologistas convidados e residentes de Dermatologia da Santa Casa de Misericórdia de Porto Alegre/UFCSPA. Devido à ausência de deslocamento e a comodidade das reuniões online, a adesão dos membros da liga foi bastante significativa.

Além dos encontros internos, a Liaderm também organizou aulas abertas à comunidade acadêmica. Dentre as atividades abertas, destaca-se a aula virtual de Introdução à Cirurgia Dermatológica, ministrada pelo dermatologista Dr. Wagner Bertolini, em julho de 2020 - Figura 2. Este encontro teve a participação de 137 estudantes de diversos estados do Brasil e até de outro país (Argentina) - Gráfico 1, advindos principalmente do curso de Medicina (126 participantes), mas também dos cursos de Enfermagem e Fisioterapia - cinco e um participantes, respectivamente.

Em relação aos meios de divulgação deste encontro virtual, a maioria dos estudantes obteve conhecimento acerca da aula a partir de redes sociais, como Instagram $\AA$ (97 participantes) e Whats $A p p \AA$ (oito participantes), além da indicação de amigos (15 participantes) e outras formas de divulgação (17 participantes).

\section{Manual 'Dermatoses Ocupacionais e COVID-19'}

Frente ao cenário da pandemia, emergiram publicações científicas destacando a importância das afecções dermatológicas na Covid-19, tanto por seus efeitos diretos - sob a forma de manifestações dermatológicas secundárias, como por seus efeitos indiretos devido ao uso de Equipamentos de Proteção Individual (EPIs) e higienização frequente.

$\mathrm{Na}$ área da saúde, a preocupação dermatológica torna-se ainda maior, visto que os profissionais de saúde necessitam fazer o uso de EPIs por períodos prolongados de jornadas de trabalho, desencadeando lesões de pele e/ou exacerbando doenças dermatológicas prévias. Nesse sentido, de forma a propiciar orientações e esclarecimentos a estes profissionais, os membros da Liaderm elaboraram, em conjunto com a docente orientadora da UFCSPA e residente de Dermatologia, um manual intitulado 'Dermatoses Ocupacionais e COVID-19' (Figura 2).

O manual abordou as lesões dermatológicas ocasionadas pelo uso de EPIs em diferentes locais do corpo, como mãos, olhos, rosto, couro cabeludo, corpo e boca, esta última contando com a colaboração de professores de Odontologia da Pontifícia Universidade Católica do Rio Grande do Sul.

Após a finalização, o manual foi submetido ao processo editorial da Editora da UFCSPA, sendo publicado no início de 2021, como parte da coleção 'Ciência, Humanidades e Covid-19'. A obra encontra-se disponível para download gratuito no site da Editora(13).

\section{Impactos da pandemia da Covid-19 nas atividades da Liaderm}

Algumas atividades da Liaderm foram profundamente prejudicadas pela pandemia da Covid-19, sem a possibilidade de amenização por meio recursos digitais. Dentre estas, a apresentação de trabalhos científicos e participação em congressos da especialidade, visto que muitos desses eventos foram cancelados ou alterados para formato virtual, assim como as atividades de extensão, que por conta do distanciamento social não puderam mais ser realizadas. Os projetos de pesquisa, cujas coletas foram finalizadas em 2019, não foram prejudicados, uma vez que se encontravam em fase de análise de dados e de redação.

\section{DISCUSSÃO}

\section{impacto da Covid-19 no ensino da dermatologia: o papel da tecnologia como alternativa educacional}

O ensino de dermatologia, na maioria das escolas médicas, apresenta poucas horas e exposição prática limitada, impactando no conhecimento e habilidades dermatológicas de médicos generalistas e especialistas de outras áreas $(14,15)$. A despeito da responsabilidade das instituições em assegurar 


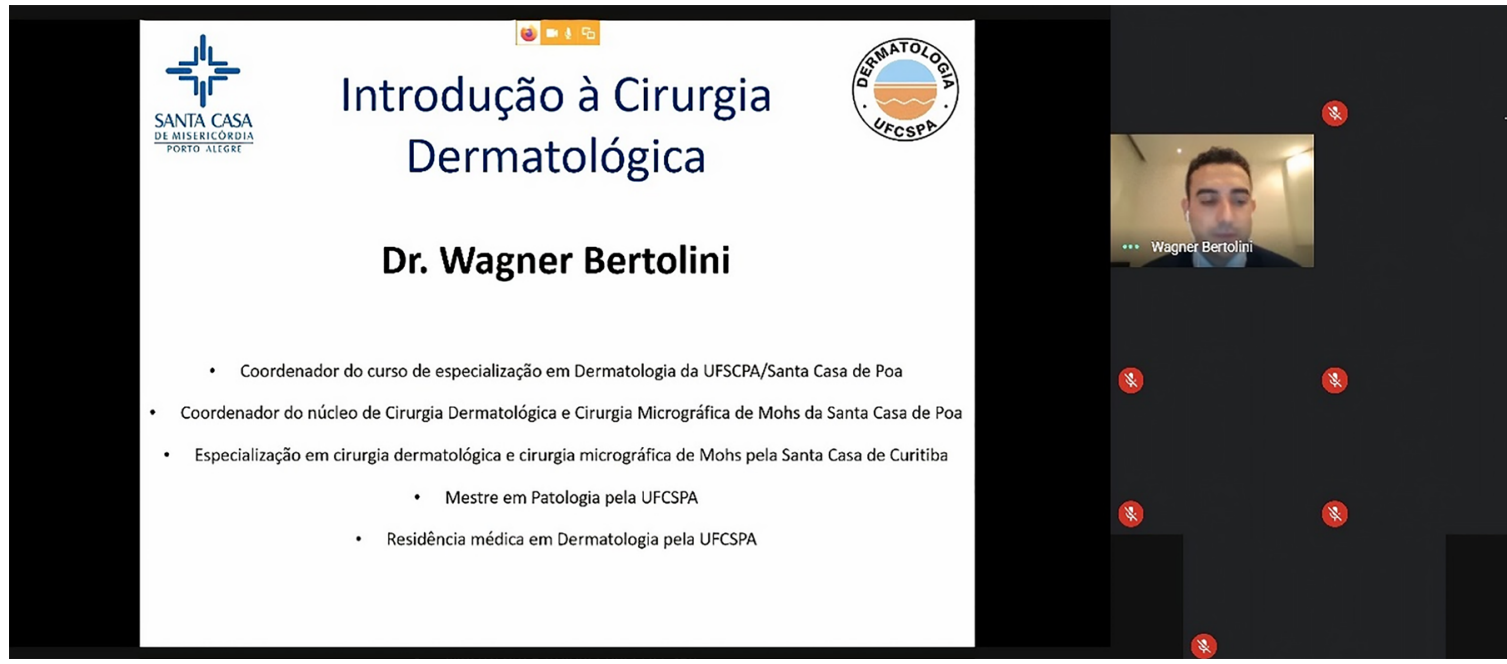

Figura 2 Aula virtual aberta da Liga Acadêmica de Dermatologia - UFCSPA, ministrada pelo Dr. Wagner Bertolini - Julho/2020.

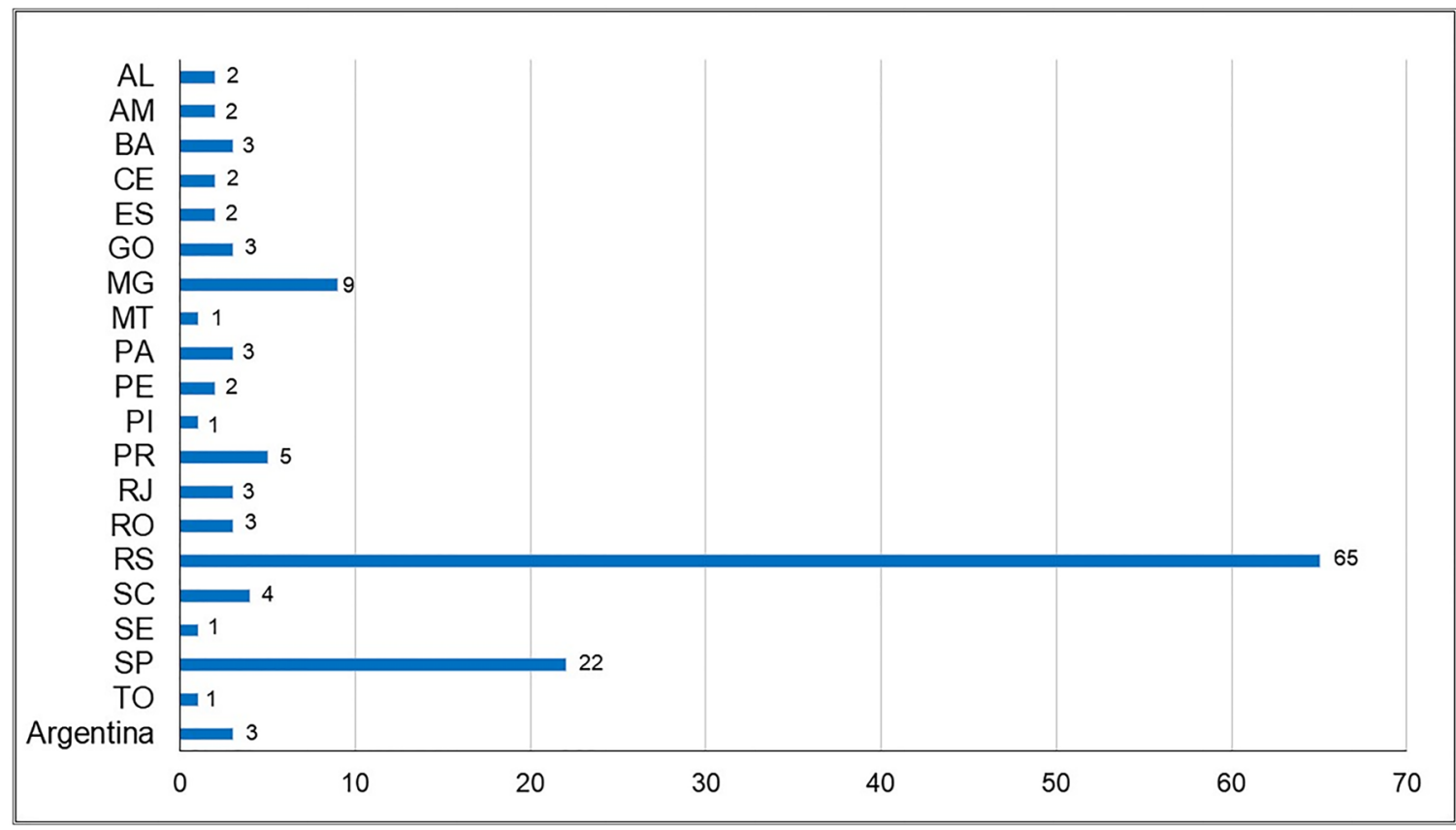

Gráfico 1 Distribuição dos participantes da aula virtual aberta da Liaderm, por estados brasileiros e país estrangeiro.

a formação médica adequada aos seus estudantes, as ligas acadêmicas (LAs) de dermatologia podem representar uma relevante forma de contato com os conteúdos e a prática da especialidade(14). Estas entidades podem contribuir com valiosas experiências de aprendizado para seus alunos membros, abordando temas da semiologia, do diagnóstico e do tratamento das doenças cutâneas, as quais afetam de forma considerável diversas condições clínicas(16).

Em meio à pandemia de Covid-19, os alunos de medicina vivenciaram um contexto atípico e sem precedentes. Segundo levantamento da Associação Brasileira de Educação Médica (ABEM), realizado em abril de 2020, cerca de $97 \%$ das escolas médicas haviam interrompido as atividades acadêmicas devido à pandemia(17). Assim como as atividades no âmbito universitário, as atividades ambulatoriais e hospitalares também foram fortemente impactadas - tendo sua carga horária reduzida ou até mesmo suspensas(5).

Neste cenário, os atendimentos dos serviços de dermatologia também foram bastante prejudicados, com uma significativa redução em suas demandas e 


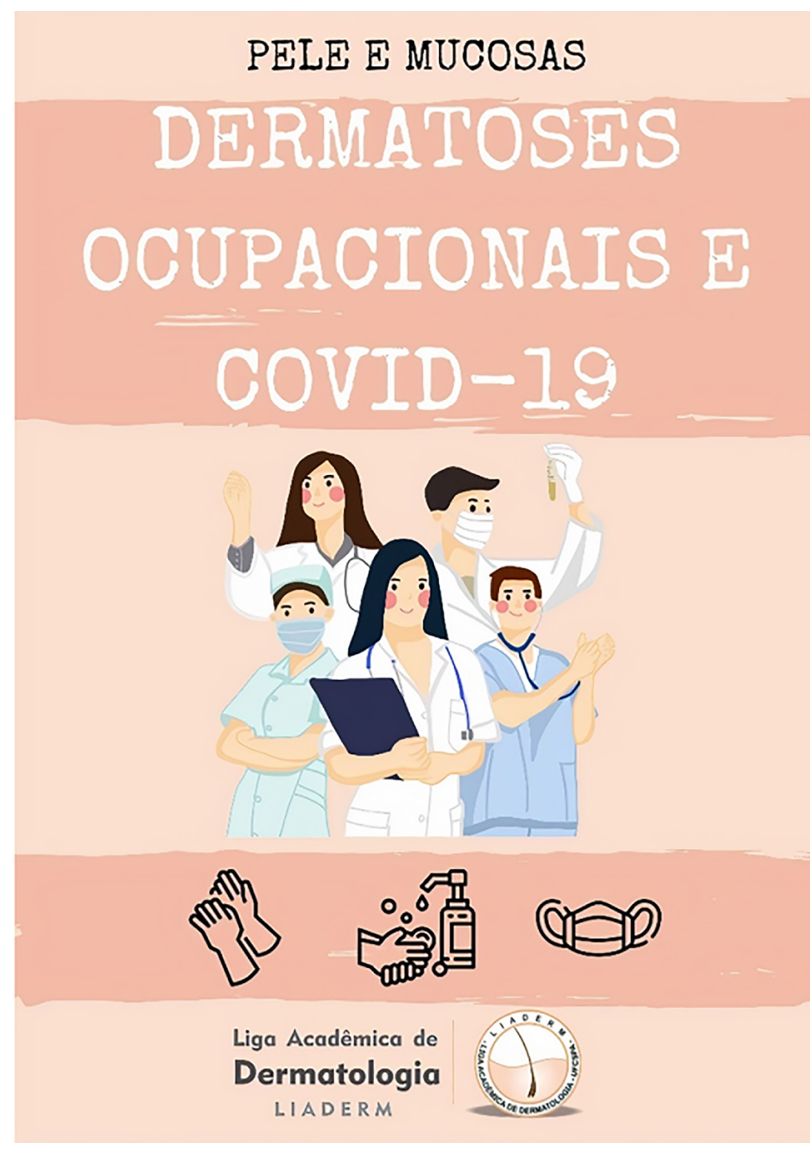

Figura 3 Capa do Manual 'Dermatoses Ocupacionais e COVID-19'. Fonte: Editora UFCSPA(13).

restrição do acesso a pacientes com quadros clínicos de maior gravidade, acarretando prejuízos assistenciais e educacionais(18). Diante deste contexto, a educação à distância em dermatologia figura-se como um recurso pedagógico estratégico para a garantia do processo de ensino e aprendizagem durante o distanciamento social imposto pela Covid-19 e a consequente interrupção das atividades nas universidades e campos de prática(4).

O uso das Tecnologias de Informação e Comunicação (TICs) tem adquirido notável relevância na prática e na educação médicas. Nessa perspectiva, emerge o conceito da 'telemedicina', definida pela OMS como a adoção de TICs como recurso de intercâmbio de informações com finalidades diagnósticas, terapêuticas, preventivas, de pesquisa e avaliação, assim como para a educação de profissionais de saúde(19). A telemedicina pode ser subdividida em duas áreas principais: a teleassistência, que possibilita o acompanhamento médico de pacientes à distância; e a teleducação ou educação à distância, modalidade que permite a educação médica continuada por videoconferências, discussões de casos clínicos, aulas teóricas online, dentre outras(20).

A educação à distância também tem alcançando importante espaço no ensino de dermatologia, presente em diversos âmbitos acadêmicos como na graduação(21), na educação permanente(22), e na residência médica(23), sendo impulsionada principalmente pelo forte componente visual ligado à especialidade. Dentre os benefícios da educação à distância em dermatologia, figura-se a capacidade de gravação e revisão de aulas e discussões, ampliação do acesso para um maior número de estudantes, oportunidade de estudo detalhado e individual das lesões cutâneas, permitindo a observação de lesões mais raras e atípica, com menor probabilidade de serem vistas nos curtos períodos de estágios curriculares. Além de vantagens como a comodidade de acesso e a prevenção das aglomerações nas salas de aula(24), medida essencial para o enfrentamento da pandemia.

Nesse sentido, Yeung et al.(25) destacam os potenciais da educação à distância no ensino de dermatologia, contribuindo com a troca de experiências e conhecimentos entre grandes audiências de estudantes, professores e residentes, assim como, com a compreensão das variantes socioeconômicas e transculturais relacionadas às doenças dermatológicas, principalmente se implementada em escala internacional. Neste relato, apresenta-se uma experiência de webmeeting sobre Cirurgia Dermatológica que registrou a participação de estudantes de medicina de diversos estados brasileiros, um exemplo ilustrativo do potencial de alcance dos recursos das TICs para a educação médica.

Sob outra perspectiva, Blakely et al.(15) e Stewart et al.(5) ressaltam a importância das sessões clínicas virtuais em pequenos grupos no ensino da dermatologia, devido a maior proximidade da interação entre estudantes e professores, oferecendo oportunidades para perguntas e de feedback com maiores níveis de confiança acerca do conhecimento em dermatologia por parte dos alunos. As atividades virtuais em pequenos grupos também foram adotadas pela Liaderm, através de suas atividades internas como discussões de casos clínicos e aulas teóricas, direcionadas apenas para seus membros. 
As ligas acadêmicas e a formação médica em dermatologia

A formação médica também pressupõe o desenvolvimento de competências humanísticas e de responsabilidade social. Sob este ângulo, as ligas acadêmicas (LAs) têm se destacado por suas ações de extensão comunitária junto à comunidade acadêmica e a sociedade $(10,26)$. A contribuição social das LAs pode ser notada a partir dessa experiência, em que os alunos membros da Liaderm elaboraram, em parceria com a professora supervisora da liga e residente de dermatologia, um manual com orientações acerca dos cuidados com a pele dos profissionais de saúde durante o período da pandemia da Covid-19.

A atuação na linha de frente do combate ao Sars-CoV-2 demanda dos profissionais o uso prolongado de Equipamentos de Proteção Individual (EPIs), tornando-os suscetíveis ao desenvolvimento de lesões cutâneas e exacerbação de doenças dermatológicas pré-existentes $(2,4)$. Dessa forma, - Manual 'Dermatoses Ocupacionais e COVID-19' elaborado pela Liaderm representou uma iniciativa pertinente para a comunidade hospitalar local.

A interação social à distância proporcionada pelas atividades da Liaderm, em meio a um período em que o distanciamento físico se fazia necessário, tornaram estas ainda mais relevantes para a coesão social e engajamento dos estudantes com o contexto universitário. Nesse sentido, Gerrard et al.(27) salientam os benefícios das atividades extracurriculares para a motivação e retenção dos alunos na universidade, bem como para o estabelecimento de relações produtivas de confiança e de suporte com tutores e professores.

Todavia, destaca-se que as ligas acadêmicas de medicina, assim como as atividades extracurriculares, em geral, não devem ocupar lacunas e/ou deficiências das universidades, tanto durante a pandemia quanto no curso regular do currículo médico. Sendo assim, considerando-se a elevada prevalência de doenças dermatológicas em diversos cenários médicoassistenciais, os cursos de medicina devem assegurar a formação adequada em dermatologia(28), de forma que as ligas de dermatologia desempenhem papel auxiliar.

Pandemia de Covid-19 e educação à distância: uma oportunidade em meio à adversidade

A conjuntura advinda da Covid-19 demandou das escolas médicas a incorporação da telemedicina e da educação à distância em seus currículos, não apenas sob perspectiva didático-pedagógica, mas também como parte do conteúdo programático. Assim, os estudantes têm a oportunidade de adquirir habilidades e competências para a adoção das TICs em suas futuras práticas profissionais(29).

Segundo Pourmand et al.(30) o ensino da telemedicina - tanto na graduação médica quanto residência médica - revela o potencial de oportunizar o aprendizado de habilidades de gerenciamento de informações, de tomada de decisões, de comunicação, assim como a valorização de aspectos éticos, regulatórios e de privacidade, intrinsecamente relacionados ao uso das TICs. Por conseguinte, os futuros profissionais tenderiam a demonstrar maior familiaridade com os recursos da telemedicina, o que poderia repercutir na oferta de assistência médica e cuidados em saúde a localidades longínquas e áreas rurais.

Vale ressaltar que, sob concepção divergente, Falcão et al.(31) pontuam a diferença entre os conceitos de ensino remoto e educação à distância. Por essa perspectiva, a educação à distância presumiria a adoção de planejamento didático e ferramentas de ensinoaprendizagem adequados, docentes com formação para o uso de TICs e suportes técnico e institucional. Sendo assim, no cenário da Covid-19, o ensino remoto se apresentaria como uma adaptação temporária frente a uma situação de crise, em que a modalidade presencial não se faz possível(20,31).

Contudo, a despeito das divergências conceituais que cercam a educação à distância, esta experiência e outras reportadas na literatura podem favorecer a reflexão e a adesão a novas estratégias educacionais e modelos assistenciais.

\section{CONSIDERAÇÕES FINAIS}

A pandemia da Covid-19 lançou grandes desafios para a educação médica, assim como para a comunidade acadêmica, instigando a reformulação de práticas pedagógicas e a adoção das Tecnologias de Informação e Comunicação (TICs) como recurso educacional. As atividades de educação à distância da Liga de Dermatologia da Universidade Federal de Ciências da Saúde de Porto Alegre (Liaderm-UFCSPA) 
alcançaram maiores públicos do que o habitual grupo de membros, representando um aprendizado para além do período de distanciamento social da pandemia.

Nessa perspectiva, a despeito das dificuldades enfrentadas neste período atípico, as inovações e soluções oriundas dessa conjuntura demonstram um importante potencial para a desconstrução de antigos paradigmas educacionais, assim como, para a consolidação das transformações curriculares - há muito tempo almejadas - pelas escolas médicas.

A contribuição das atividades extracurriculares na formação em Medicina, especialmente das ligas acadêmicas, revela-se de forma mais clara a partir desta experiência e de outras similares, evidenciando a importância do estímulo da autonomia, proatividade e engajamento dos estudantes no contexto universitário.

\section{REFERÊNCIAS}

1. Sun $P, \operatorname{Lu} X, X u$ C, Sun $W$, Pan B. Understanding of COVID-19 based on current evidence. J Med Virol. 2020;92(6):548-51.

2. Darlenski R, Tsankov N. COVID-19 pandemic and the skin: what should dermatologists know? Clin Dermatol. 2020; (xxxx):4-6.

3. OPAS OP-A da S-. OPAS/OMS Brasil - Folha informativa - COVID-19 (doença causada pelo novo coronavírus) [Internet]. 2020 [citado 21 de julho de 2020]. Disponível em: https://www.paho.org/bra/index.php?option=com content\&view $=$ article\&id $=6101$ : covid $19 \&$ Itemid $=875$

4. Wollina U. Challenges of Covid-19 Pandemic for Dermatology. Dermatol Ther. 2020;(March):1-5.

5. Stewart CR, Chernoff KA, Wildman HF, Lipner SR. Recommendations for medical student preparedness and equity for dermatology residency applications during the COVID-19 pandemic. J Am Acad Dermatol. maio de 2020;

6. Reinholz M, French LE. Medical education and care in dermatology during the SARS-CoV2 pandemia: challenges and chances. J Eur Acad Dermatology Venereol. 2020;34(5):e214-6.

7. Behar PA. Competencias em Educacao a Distância. $1^{\text {a }}$. Behar PA, organizador. Porto Alegre: Editora Penso; 2013. 308 p.

8. Singh DG, Boudville N, Corderoy R, Ralston S, Tait CP. Impact on the dermatology educational experience of medical students with the introduction of online teaching support modules to help address the reduction in clinical teaching. Australas J Dermatol. 2011;52:264-9.

9. Chehuen Neto JA, Sirimarco MT, Cândido TC, Ferreira I de A, Cambraia R, Campos F, et al. Students- perspectives on the parallel curriculum in medical schools. Rev Médica Minas Gerais. 2013;23(4):467-78.

10. Ferreira IG, Carreira LB, Botelho NM, Souza LEA de. Atividades extracurriculares e formação médica: diversidade e flexibilidade curricular. Interdiscip J Heal Educ. $2016 ; 1(2): 114-24$.

11. Ferreira IG, Carreira LB, Murphy N, Soares ACB, Fonseca PCC, Sousa LEA de. Extracurricular activities: a comparative perspective among health colleges in Brazil and Ireland. ABCS Heal Sci. 2 de agosto de 2018;43(2):97-103.

12. Hamamoto Filho PT. Como as ligas acadêmicas podem contribuir para a formação médica ? Diagnóstico e Trat. 2011;16(3):137-8.

13. Editora UFCSPA. Obras publicadas da Editora da Universidade Federal de Ciências da Saúde de Porto Alegre [Internet]. Coleção UFCSPA - Ciência, Humanidades e Covid-19 / Dermatoses Ocupacionais e Covid-19. 2021 [citado 11 de setembro de 2021]. Disponível em: https://www.ufcspa.edu.br/vida-nocampus/editora-da-ufcspa/obras-publicadas

14. Ulman CA, Binder SB, Borges NJ. Assessment of medical students' proficiency in dermatology: Are medical students adequately prepared to diagnose and treat common dermatologic conditions in the United States? J Educ Eval Health Prof. 17 de maio de 2015;12:18.

15. Blakely K, Bahrani B, Doiron P, Dahlke E. Early Introduction of Dermatology Clinical Skills in Medical Training. J Cutan Med Surg. 15 de janeiro de 2020;24(1):47-54.

16. Kirshen C, Shoimer I, Wismer J, DesGroseilliers JP, Lui $\mathrm{H}$. Teaching dermatology to canadian undergraduate medical students. J Cutan Med Surg. 2011;15(3):150-6.

17. Associação Brasileira de Educação Médica. Resultados preliminares da análise dos questionários de estudantes, professores e gestores de escolas médicas. Brasília; 2020.

18. Ferreira IG, Weber MB. El día después: el impacto de la pandemia COVID-19 en la residencia de Dermatología. Actas Dermosifiliogr. novembro de 2020;1(1):1-6.

19. World Health Organization W. Telemedicine: opportunities and developments in Member States: report on the second global survey on eHealth 2009. Genebra; 2010.

20. Soirefmann M, Blom MB, Leopoldo L, Cestari TF. Telemedicina: uma revisão da literatura. Rev HCPA Fac Med Univ Fed Rio Gd do Sul. 2008;28(2):116-9.

21. Wagner $\mathrm{R}$, Jalalat $\mathrm{S}$. Utility of a dermatology interest group blog: the impact of medical student interest groups and Web 2.0 tools as educational resources. Adv Med Educ Pract. setembro de 2014;5:331-7.

22. Goiriz R, Butler C, Rajpopat S, Sahota A, Hubbard V, Jolliffe V. Perceived benefits following completion of a clinical dermatology online programme for general practitioners. Educ Prim Care. 2016;27(4):335-7.

23. Wanat $K A$, Newman S, Finney $K M$, Kovarik $C L$, Lee I. Teledermatology Education: Current Use of 
Teledermatology in US Residency Programs. J Grad Med Educ. $2016 ; 8(2): 286-7$.

24. Dolev JC, O'Sullivan $P$, Berger T. The eDerm online curriculum: A randomized study of effective skin cancer teaching to medical students. J Am Acad Dermatol. 2011;65(6):e165-71.

25. Yeung $H$, Sargen MR, Luk KM, Berry EG, Gurnee EA, Heuring $E$, et al. Teledermatology and teledermatopathology as educational tools for international dermatology: a virtual grand rounds pilot curriculum. Int J Dermatol. 2018;57(11):1358-62.

26. Costa BEP, Hentschke MR, Silva ACC, Barros A, Salerno $M$, Poli-de-Figueiredo $C E$, et al. Reflexões sobre a importância do currículo informal do estudante de medicina. Sci Med (Porto Alegre). 2012;22(3):162-8.

27. Gerrard S, Billington J. The perceived benefits of belonging to an extra curricular group within a pre- registration nursing course. Nurse Educ Pract. maio de 2014;14(3):253-8.

28. Waller BAM, Liu A, Fleming P, Lansang P. Undergraduate Dermatology Medical Education: Results of a LargeScale Patient Viewing Program. J Cutan Med Surg. 2019;23(5):482-7.

29. Jumreornvong O, Yang E, Race J, Appel J. Telemedicine and Medical Education in the Age of COVID-19. Acad Med. 1 de dezembro de 2020;95(12):1838-43.

30. Pourmand A, Ghassemi M, Sumon K, Amini SB, Hood C, Sikka N. Lack of Telemedicine Training in Academic Medicine: Are We Preparing the Next Generation? Telemed e-Health. 2021;27(1):62-7.

31. Falcão MC, Fonseca CDPP, Danti GV. Ensino na residência médica em tempos de Covid-19. Rev Paul Pediatr. 2020;38:2-4. 
A experiência de liga acadêmica de dermatologia na pandemia da Covid-19

Autor correspondente:

Iago Gonçalves Ferreira

E-mail: iago_goncalves14@hotmail.com

Editor:

Prof. Dr. Marcelo Riberto

Recebido: $21 / 08 / 2020$

Aprovado: 05/05/2021 